\title{
TECNICAS E INSTRUMENTOS PARA RECOGER DATOS DEL HECHO SOCIAL EDUCATIVO
}

\section{TECHNIQUES AND TOOLS TO COLLECT DATA FROM THE EDUCATIONAL SOCIAL FACT}

\author{
Marcelo Remigio Castillo Bustos ${ }^{1}$
}

Recibido: 2020-09- 26 / Revisado: 2020-10-12 / Aceptado: 2020-11-01 / Publicado: 2021-01-01

Forma sugerida de citar: Castillo-Bustos, M. R. (2021). Técnicas e instrumentos para recoger datos del hecho social educativo. Retos de la Ciencia. 5(10), pp. 50-61. https://doi.org/10.53877/rc.5.10.20210101.05

\section{RESUMEN}

Este trabajo presenta reflexiones acerca del uso de las técnicas e instrumentos empleados para recoger datos del hecho social educativo. Este se entiende como un hecho social en su más amplia significación y sentido. La investigación se desarrolló enmarcada en el paradigma cualitativo, por lo que, se recurrió a los métodos de análisis, síntesis, hermenéutico, holístico y dialéctico, con la finalidad de comprender el objeto de estudio y posibilitar una mejor aplicación de técnicas e instrumentos en el desarrollo de investigaciones contextualizadas en el campo socioeducativo. Se revela que a pesar de haber varios enfoques epistemológicos que orientan la construcción del conocimiento, la investigación cualitativa emerge como la única posibilidad para concretar tal propósito, a partir de la experiencia vivida del investigador en la realidad investigada. De ahí que, debe tenerse en cuenta la naturaleza de la investigación, el objeto de estudio, el marco de referencia inicial, el contexto en el cual se investiga, los objetivos y el diseño metodológico que regirán el proceso investigativo, para seleccionar las técnicas e instrumentos de recogida de datos en el hecho social educativo, ya que este, está dinamizado por las relaciones e interrelaciones entre seres humanos.

Palabras clave: hecho social educativo, técnicas, instrumentos, datos.

\section{ABSTRACT}

This paper presents reflections about the use of techniques and tools used to collect data from the educational social fact, which is understood as a social fact in its broadest meaning and sense. The research was developed framed in the qualitative paradigm, reason why are used the methods of analysis, synthesis, hermeneutics, holistic and dialectic. This has been done with the purpose of understanding the object of study and enabling a better application of techniques and instruments in the development of contextualized researches in the socio-educational field. It is revealed that despite having several epistemological approaches that guide the construction of knowledge, qualitative research emerges as the only possibility to achieve this

\footnotetext{
${ }^{1}$ Doctor en Ciencias Pedagógicas (Ph.D). Profesor titular en la Facultad de Filosofía, Letras y Ciencias de la Educación de la Universidad Central del Ecuador. Ecuador. Email: mrcastillob@uce.edu.ec / ORCID: https://orcid.org/0000-0002-2615-7482
} 
purpose, based on the lived experience of the researcher in the reality investigated. Therefore, the nature of the research, the object of study, the initial reference frame, the context in which it is being investigated, the objectives and the methodological design that will govern the research process must be taken into account, in order to select the techniques and instruments of the data compilation in the educational social fact since it is energized by the relationships and interrelationships between human beings.

Keywords: educational social facts, techniques, instruments, data.

\section{INTRODUCCIÓN}

La investigación científica es un proceso ordenado y sistemático de indagación, que, mediante la aplicación de métodos, técnicas, instrumentos y demás posibilidades, viabiliza el estudio de la realidad. De esta forma, construye un conocimiento válido para la continua adaptación del ser humano a los cambios y transformaciones que experimenta el mundo en todas sus esferas. Además, ella está orientada por la especificidad de sus enfoques epistemológicos. En tal sentido, la investigación cualitativa, conocida también como investigación social; por su carácter experiencialista-vivencialista, emerge como la alternativa epistemológica idónea para abordar los hechos o fenómenos sociales, entre los cuales está inmerso el hecho social educativo, debido a que este, se encuentra dinamizado por las relaciones e interrelaciones entre seres humanos en función del desarrollo y la transformación sociocultural individual y colectiva.

El hecho social educativo, por su naturaleza, se diferencia significativamente de otros hechos sociales, debido a sus implicaciones en el desarrollo y transformación sociocultural, a partir de la formación de seres humanos. La educación conlleva a la socialización del ser, basada en normas, valores y principios, todo lo cual, orienta los roles individuales y colectivos que los seres humanos cumplen y cumplirán en la sociedad. Teniendo en cuanta que actualmente se vive un cambio de época, caracterizado por verdaderas revoluciones a nivel social, político, tecnológico, científico, etc., las personas que asumen la tarea de educar deben estar preparadas para enfrentar todas situaciones de relacionadas con tales cambios, caso contrario, el hecho social educativo seguirá realizándose con pocos niveles de contextualización, y tendrá un limitado impacto en el desarrollo y transformación sociocultural individual y colectivo.

A pesar de que la investigación cualitativa ha recibido amplias críticas $u$ objeciones desde otros enfoques epistemológicos, debido a la ambigüedad de sus métodos; no es menos cierto, que constituye la única alternativa o posibilidad epistemológica para comprender e interpretar los hechos, fenómenos o situaciones relacionados con los modos o maneras de ser, sentir, pensar y actuar de las personas en su contexto natural, o cuando se trata de comprender la realidad objeto de estudio a través de vivir las experiencias que los otros viven. De ahí la importancia, de continuar promoviendo debates científicos, acerca de sus concepciones y prácticas, ya que estas tienen significativa incidencia en el momento de desarrollar el quehacer investigativo y sobre todo cuando se trata de emitir resultados (conocimientos) desde su perspectiva.

En tal sentido, la elección de las técnicas e instrumentos más adecuados para recoger datos del hecho social educativo, de manera similar a cualquier otro estudio dentro del campo de las Ciencias Sociales, constituye un proceso de gran 
responsabilidad para el investigador, teniendo en consideración que la pertinencia de las técnicas y la calidad de los instrumentos aplicados, determinarán en cierta medida la calidad de los datos recogidos. A pesar de haber una clara diferenciación entre investigadores de los distintos enfoques epistemológicos, debe reconocerse que "la selección de técnicas generalmente es independiente del enfoque epistemológico del investigador". (Campoy y Gomes, 2011, p. 275) por consiguiente, se precisa la necesidad de elegir apropiadamente las técnicas e instrumentos, para recoger los datos suficientes del objeto de estudio y elevar resultados investigativos válidos 0 plausibles.

Para facilitar una mayor comprensión acerca del hecho social educativo, se recurre al planteamiento de Durkheim (2001), quien acuña como un concepto básico en la sociología y la antropología, el hecho social, refiriéndose a todo comportamiento o idea presente en un grupo social, sea este respetado y compartido o no por los individuos, cuando señala: Un hecho social es toda manera de hacer, establecida o no, susceptible de ejercer sobre el individuo una coacción exterior; o también, el que es general en la extensión de una sociedad determinada teniendo al mismo tiempo una existencia propia, independiente de sus manifestaciones individuales" (p. 52).

Los hechos sociales constituyen maneras o modos de obrar, pensar y sentir exteriores al sujeto, pero que conllevan a hacer algo debido a su poder imperativo y coercitivo. Entonces, los hechos sociales son impuestos a los individuos, quienes tienen la obligación de hacer algo, estando o no de acuerdo; nótese que los hechos sociales, tienen un sustrato en la sociedad. Por tanto, el ser humano toma una posición de poco cuestionamiento a una "doctrina moral, o sea, la imposición de la legitimidad de una determinada cultura o forma de vida" (Usategui, 2004, p. 11), en este caso a través del hecho social educativo, en sus distintas maneras o modos de concretarse.

Hay que reconocer que existe una clara diferencia entre los fenómenos psíquicos y la exterioridad de los hechos sociales. "Los hechos sociales se diferencian de los hechos psíquicos no solo en calidad: tienen otro sustrato, no evolucionan en el mismo medio, no dependen de las mismas condiciones" (Durkheim, 1895, p. 22), es decir, los fenómenos psíquicos refieren a los cambios o modificaciones que se dan en la mente o psiquis del ser humano y tienen lugar en el Yo; mientras que los hechos sociales, son costumbres o creencias que se dan en la sociedad. Para que exista 0 se realice un hecho social, varias personas deben haber combinado sus acciones en torno a algún hecho, dando lugar a algo nuevo. Por consiguiente, ningún hecho social puede ser entendido a partir de la individualidad del sujeto. Durkheim, se refiere también al concepto de institución, como todas las creencias y modos de conducta instituidas por la colectividad o grupo de personas, lo cual, hace que la sociología emerja como la ciencia de las instituciones, de su origen y su funcionamiento.

Sobre las bases de los fundamentos expuestos, se reconoce que el hecho social educativo se da en distintos procesos institucionalizados premeditadamente por la sociedad, para promover intencional, organizada y sistemáticamente la formación integral del ser humano, en cuyo proceso los planteles educativos cumplen un rol fundamental, al constituirse en los espacios de encuentro entre docentes, estudiantes y demás actores educativos en función de un necesario intercambio de experiencias, conocimientos, valores y valoraciones, como esencia del desarrollo y la transformación socio-cultural individual y colectiva. Las personas en sus distintas etapas de desarrollo tienen la obligación de cursar ciertos niveles educativos, para cumplir con la imposición social de ser educados formalmente (educación 
escolarizada), lo cual, constituye requisito obligatorio para el acceso a otras posibilidades sociales y laborales.

El hecho social educativo, sin limitarse a un nivel de estudios o modalidad en particular, constituye un espacio con amplias posibilidades para la investigación social, debido a la multiplicidad de hechos y fenómenos que en este se desarrollan. En tal sentido, la investigación cualitativa encuentra en el hecho social educativo, el lugar propicio para constituirse en el eje dinamizador de la acción pedagógica, que condiciona la realización de los procesos complejos implicados en el desarrollo y la transformación de la cultura, a partir de una educación de calidad y calidez, en función de las exigencias de una sociedad que cambia y se transforma permanentemente. Cabe advertir que "el mundo es algo que fluye continuamente, es decir no puede ser pensado como algo fijo o estático. La realidad siempre remite a un proceso, a un desarrollo en el tiempo-historia, a una tradición que nosotros retomamos". (Gurdián, 2010, p. 163)

Al amparo de los argumentos expuesto en los párrafos anteriores, el presente trabajo conlleva a un análisis sobre del concepto, la importancia y la utilidad de las técnicas e instrumentos para recoger datos del hecho social educativo, con la finalidad de ampliar la visión acerca de la investigación cualitativa y su aplicación pertinente y contextualizada en el campo de la educación, teniendo en cuenta que en este campo, se realiza el hecho social educativo como un fenómeno social en su más amplia significación y sentido. El mismo que se encuentra dinamizado por las relaciones e interrelaciones entre seres humanos, donde todos sus participantes interactúan conscientemente de acuerdo con sus intereses y motivaciones.

\section{DESARROLLO}

\section{Técnicas e instrumentos de recolección de datos}

En toda investigación y particularmente en la investigación cualitativa, es necesario llevar a cabo la recolección de datos, lo cual, es clave para el éxito de la labor investigativa. Cabe recalcar que el investigador tiene la tarea compleja de elegir las técnicas e instrumentos de recolección de datos más idóneos, por lo que requiere de cierta experticia en tal proceso. La recolección de datos, más entendida como la medición, es considerada una condición fundamental para la construcción de un conocimiento científico válido o plausible, en función de la realidad contextual. Se asume entonces, que un dato es un concepto que expresa una abstracción del mundo real, de lo sensorial, susceptible de ser percibido por los sentidos, directa 0 indirectamente. En el caso de la investigación cualitativa, los datos son adquiridos o recolectados a través de la vivencia o experiencia vivida del investigador en el entorno natural, es decir, en el lugar donde se producen los hechos, situaciones o fenómenos objeto de investigación.

Varios investigadores coinciden en que la recolección de datos refiere al uso de técnicas e instrumentos de recopilación de información acerca el objeto de estudio, para responder a las preguntas de investigación, sobre las bases del análisis e interpretación correspondientemente. Teniendo en cuenta que la dinámica de los fenómenos sociales en la actualidad es ampliamente cambiante, se hace necesario reflexionar constantemente en torno a la pertinencia de las técnicas e instrumentos que se utilizan para recoger los datos, más aún cuando desde la perspectiva de la investigación cualitativa, cada hecho o situación debe ser visto por el investigador como si estuviera ocurriendo por primera vez o como si fuera único en el universo. Si 
los hechos y situaciones sociales son tan diversos, como diversos son los seres humanos, es propio pensar que las técnicas e instrumentos de recolección de datos, presentan limitaciones frente a la diversidad de los hechos sociales.

Teniendo en cuenta que la investigación cualitativa se desarrolla a partir de la observación directa a las situaciones o fenómenos de estudio o desde la experiencia vivida del investigador en el lugar de los hechos; el proceso cualitativo no es de carácter lineal como la investigación cuantitativa, sus etapas esencialmente emergen como las acciones específicas que se desarrollan para cumplir con los objetivos de la investigación y dar respuesta a las preguntas del estudio. El investigador, empieza a recolectar e incluso a analizar los datos, desde el mismo momento en que toma contacto con la realidad investigada, o por el simple hecho de observar lo que en el contexto investigado ocurre; lo cual, constituye una peculiaridad de la referida investigación. Por consiguiente, la investigación cualitativa tiene significativa importancia, al realizarse en el campo de la educación, puesto que esta, como seña Casilimas (1996) "se convierte así en una producción colectiva donde el maestro tiene que negociar el conocimiento para mantener el control del proceso" (p. 283).

Las técnicas de recolección de datos son concebidas como el conjunto de procedimientos e instrumentos empleados para orientar, recoger, conservar, ordenar y transmitir los datos, es decir, para desarrollar los sistemas de información inherentes al proceso investigativo. Teniendo en cuenta la existencia de una diversidad de técnicas de recolección de datos, el investigador, a partir de valorar la naturaleza del objeto de investigación, empleará las técnicas más adecuadas y pertinentes. Cabe señalar, que actualmente se observa una amplia flexibilidad en cuanto a la elección y aplicación de técnicas de recolección de datos, sin discriminar el enfoque epistemológico al que estas tradicionalmente se correspondan, lo cual, se sustenta en la necesidad de obtener datos suficientemente precisos para arribar a conclusiones en cierto modo, objetivas.

En cuanto a los instrumentos de recolección de datos, se puede decir que constituyen los recursos o medios utilizados para obtener y archivar los datos requeridos sobre el objeto de estudio, registrándolos de manera organizada en función de los objetivos de la investigación. En el caso de la investigación cualitativa, debe tenerse en cuenta que el ser humano es un ser social por esencia, es decir, su realización integral está mediada por las relaciones e interrelaciones con los demás, por lo que los hechos, situaciones y/o fenómenos en los cuales está inmerso, son cambiantes de acuerdo con su desarrollo histórico, por lo que, debe otorgarse especial atención a los cambios y transformaciones que experimenta a lo largo de la vida.

\section{Aspectos que debe tener en cuenta el investigador cualitativo para seleccionar las técnicas de recogida de información}

Según Tejedor (1986) "la investigación cualitativa, naturalista o etnográfica requiere una metodología sensible a las diferencias, a los procesos singulares y anómalos, a los acontecimientos imprevisibles, al cambio, a las observaciones manifestables y a los significados latentes" (p. 88), por tanto, como se señaló en líneas anteriores, la selección de técnicas e instrumentos de recolección de datos, constituye una delicada tarea para el investigador cualitativo, ya que de la calidad de las referidas técnicas e instrumentos, dependerá en cierta medida de la calidad de la información que recoja y consecuentemente, los resultados de la investigación. Por consiguiente, el investigador cualitativo requiere de experiencia para seleccionar adecuadamente 
las técnicas y diseñar los instrumentos de recogida de datos necesarios y suficientes para arribar a conclusiones.

Hay acuerdos en el sentido que, al momento de seleccionar las técnicas e instrumentos de recolección de datos, el investigador cualitativo, debe tener en cuenta aspectos relacionados con los principales elementos de la investigación; y que ello, constituye una de las tareas más relevantes del proceso investigativo, por su significativa influencia en los resultados de la investigación. Entre los principales aspectos a considerar se mencionan los siguientes: La naturaleza del estudio, el tipo de problema u objeto de investigación, la definición de las unidades de análisis, el tipo y la confiabilidad de las fuentes de donde se obtendrán los datos, el universo de estudio, el tamaño y tipo de muestra, los recursos disponibles y la coyuntura u oportunidad para realizar el estudio.

Hay que considerar que el hecho social educativo, se realiza en torno a la formación del ser humano, lo cual imprime mayor responsabilidad y compromiso del investigador, debido a la peculiaridad del contexto en el que se realiza el referido objeto de investigación.

Se precisa que la aplicación de técnicas e instrumentos de recolección de datos incide significativamente en la totalidad del proceso investigativo y más todavía en los resultados de la investigación. Campoy y Gomes (2011), plantean que la las técnicas cualitativas tienen las siguientes ventajas: a) permiten abordar problemas complejos como son el estudio de creencias, motivaciones o actitudes de la población, b) posibilitan la participación de individuos con experiencias diversas, lo cual permite tener una visión más amplia de los problemas, c) permiten la generación de un gran número de ideas de forma rápida, y disminuye el tiempo para la toma de decisiones, d) su realización suele ser sencilla, no requiriendo complicados diseños ni complicadas pruebas estadísticas; y, e) tienen un bajo coste económico, y las siguientes desventajas: a) carecen de la precisión de las técnicas cuantitativas, b) sus conclusiones no son generalizables.

Los autores referidos en el párrafo anterior al señalar que las técnicas cualitativas permiten abordar problemas complejos como creencias, motivaciones o actitudes de la población; destacan que el hecho social en su esencia debe ser visto de manera holística y compleja, es decir, inductivamente en su más amplia significación y sentido. Si bien, en la investigación cualitativa la participación de individuos con experiencias diversas permite tener una visión más amplia de los problemas, no es menos cierto, que debe tenerse en cuenta que cada hecho, situación u objeto de estudio es único o auténtico en el contexto investigado, de ahí que la visión de los propios investigados aporta inconmensurablemente en la calidad de datos obtenidos. Téngase en cuenta que los referidos actores "ayudan a comprender la visión que los tomadores de la decisión tienen del mundo que enfrentan" (López y Salas, 2019, p. 15), lo cual, permite la generación de un gran número de ideas de forma rápida y disminuye el tiempo para la toma de decisiones, situación que es más difícil desde otro tipo de investigación.

Campoy y Gomes (2011), al referirse a que la realización de las técnicas cualitativas, "suele ser sencilla, no requiriendo complicados diseños ni complicadas pruebas estadísticas" y que "tienen un bajo coste económico" (p. 277); están considerando limitadamente el carácter inductivo de la investigación cualitativa, puesto que, en este tipo de investigación cada hecho, situación o fenómeno de estudio al ser visto como único en el universo, exige del investigador cierta experiencia en los distintos procesos implicados en el sistema de información. 
Asimismo, si cada objeto de investigación es distinto con respecto a otros, aún en el mismo contexto, se requerirá tantas investigaciones como objetos (hechos sociales) existen, por lo que, producir conocimiento desde la investigación cualitativa, implica una inversión elevada, en términos de recursos económicos, materiales y tiempo para cumplir de técnicamente con los procesos exige.

Por otra parte, los mismos autores al señalar que las técnicas cualitativas "carecen de la precisión de las técnicas cuantitativas" y que sus "conclusiones no son generalizables", poco consideran que "el énfasis colocado solo en el rigor metodológico enmascara la ausencia de una elaboración teórica; porque creemos además que la metodología no es solo cuestión de técnicas sino que está íntimamente relacionada con posturas filosóficas" (Schettini y Cortazzo,2015, p. 15) de quienes participan en el proceso investigativo. Por consiguiente, el hecho social educativo y su esencia, será abordado con pertinencia desde la investigación cualitativa, sus técnicas, instrumentos y demás posibilidades vinculadas con el ser humano y sus relaciones e interrelaciones.

\section{Aspectos que debe tener en cuenta el investigador cualitativo al seleccionar/diseñar los instrumentos para recoger datos}

Siendo los instrumentos, las principales herramientas empleadas en el trabajo de campo, que, a su vez, es el camino hacia la obtención de los datos de la realidad objeto de estudio, y teniendo en cuenta que la investigación cualitativa es fundamentalmente inductiva, los investigadores desarrollan conceptos, intelecciones, comprensiones e interpretaciones de la realidad, más no, explicaciones de esta. Los hechos y/o situaciones investigadas, desde una perspectiva holística son vistos como un todo, por tanto, las personas y los escenarios no son reductibles a variables. Es decir, cada hecho, situación o fenómeno es visto por el investigador como si estuviera sucediendo por primera vez en el universo, de ahí, que la investigación cualitativa trabaja con categorías de análisis, las mismas que han sido explicadas por varios autores como atributos o características manifiestas de un objeto o fenómeno.

Desde esa perspectiva, las categorías de análisis constituyen conceptos que tienen un significado, el mismo que puede estar relacionado con situaciones, problemas, contextos, opiniones, acontecimientos, sentimientos y relaciones interpersonales, entre otros aspectos inherentes al ser humano. Cada categoría de análisis representa un concepto empleado en el proceso investigativo para ir respondiendo progresivamente a la pregunta de investigación. De ahí, se asume que las categorías de análisis emergen inicialmente de los propósitos y del marco de referencia de la investigación, pero existe la posibilidad que surjan otras categorías de análisis, a partir de la aplicación de los instrumentos y del correspondiente análisis de los datos ya obtenidos.

Téngase en cuenta que las técnicas y los instrumentos de recolección de datos sintetizan la etapa de planeación de la investigación, de ahí que, el investigador cualitativo al momento de escoger las técnicas y diseñar los referidos instrumentos, requiere básicamente tener en cuenta los siguientes aspectos: la naturaleza de la investigación, el objeto del estudio, el marco de referencia inicial, el contexto en el cual se investiga, los objetivos y el diseño metodológico. Caso contrario, se corre el riesgo de escoger técnicas inadecuadas y diseñar instrumentos que no se correspondan al objeto de estudio.

En cuanto a la naturaleza de la investigación cualitativa al abordad el hecho social educativo, debe mantener su característica fundamental, como es la capacidad del investigador para concretar su estudio a partir de la experiencia vivida, es decir, los 
hechos, acciones, normas, valores y demás situaciones sociales que configuran el objeto de investigación, deben ser abordados desde la perspectiva de los involucrados y en función del contexto en el que están siendo estudiados. Por consiguiente, como afirma Mella (1998), refiriéndose a los investigadores "el carácter reflexivo de la investigación social, vale decir reconocer que somos parte del mundo social que estudiamos" (p. 4).

Se destaca que para la investigación cualitativa no existen hechos, personas y/o escenarios que no merezcan la atención del investigador o que no requieran ser investigados, por consiguientes, las opiniones y perspectivas de las personas son de invaluable importancia, debido a que, desde la configuración de la referida investigación, se busca comprender e interpretar la realidad en su esencia, más no explicarla. Todo lo cual tiene base en un amplio sentido humanístico de los métodos cualitativos, los mismos que no están hechos para reducir las opiniones y comportamientos de las personas a expresiones estadísticas o cuestiones medicionales, ya que con ello se pierde la esencia de la investigación social centrada en la riqueza de la condición humana, lo cual solo es posible captar a través de la experiencia vivida naturalmente en el lugar donde se producen los hechos y situaciones sociales.

Teniendo en cuenta que la investigación cualitativa básicamente se constituye en una aproximación sistémica orientada a la descripción de las experiencias de la vida para darles un significado, y que la misma tiene como objeto de estudio los acontecimientos, acciones, normas, valores y demás posibilidades inherentes a las relaciones e interrelaciones entre seres humanos; se precisa, que no hay que perder de vista las condiciones diversas presentes en el hecho social educativo, en dependencia de la realidad contextual en la que se concreta.

En lo que tiene que ver con el marco de referencia inicial, es apropiado señalar que en la investigación cualitativa no se cuenta o no se tiene un marco teórico, pues cada hecho situación o fenómeno objeto de estudio, es visto por el investigador como si estuviera sucediendo por primera vez en el universo, por lo que en suma podría tener un marco referencial. Lógicamente, el hecho social educativo a pesar de desarrollarse de manera similar en distintos contextos no es menos cierto, que se realiza de acuerdo con los intereses, motivaciones y acciones de las personas que en el mismo interactúan; es decir, no habrá de ninguna manera dos hechos sociales educativos iguales. "El investigador cualitativo cree que los seres humanos son esencialmente distintos y por lo tanto no pueden ser encasillados en categorías únicas". (Arroyo, 2006, p. 5) o en marco teórico alguno.

La investigación cualitativa en relación con el contexto en el que se desarrolla tiene la particularidad que el investigador se acerca a la realidad de manera natural, vivir lo que los investigados viven y sentir lo que estos sienten, como mecanismos que conllevan a la comprensión e interpretación de los hechos, situaciones y/o fenómenos sociales desde la perspectiva de la o las personas que conforman su objeto de estudio, o simplemente desde la perspectiva de los investigados. De ahí que, al momento de abordar el hecho social educativo como objeto de investigación, sin lugar a dudas, se tendrá exigencias inconmensurables relacionadas con los modos de ser, sentir, pensar y actuar de seres humanos, mismos que al igual que el resto, se encuentran en constante proceso de desarrollo y transformación.

En torno a los objetivos de la investigación, a partir del análisis se revela la existencia de una diversidad de criterios, no obstante, en función de este trabajo, se 
hace referencia a los planteamientos de Martínez (2014), quien, desde su experiencia, señala:

En las investigaciones cualitativas se fijan unos objetivos a lograr: Algunos son más bien general y otros específicos, pero todos deben ser relevantes para las personas interesadas en la investigación. A veces, es preferible fijar sólo objetivos generales y determinar los específicos durante la marcha, para no buscar metas que quizá resulten triviales o imposibles (pp. 130-131).

En cuanto a los objetivos de investigaciones relacionadas con el hecho social educativo, si bien puede estar orientado a clarificar los fenómenos propios de las interacciones entre los intervinientes en el contexto socioeducativo, se precisa que el ser humano aprende y se desarrolla en todo momento y espacio; por tanto, las especificidades (objetivos específicos) emergerán en la medida que se desarrolle la investigación, más no en sentido contrario. Los objetivos específicos de la investigación cualitativa y particularmente, cuando esta se aplica en el hecho social educativo, deben ser planteados cuando la investigación haya alcanzado el nivel suficiente para visualizar las verdaderas características del objeto, en función de la realidad contextual en la cual se investiga.

En relación con los objetivos, varios autores entre ellos Martínez (2006), coinciden en que:

En las investigaciones cualitativas se fijan unos objetivos a lograr: Algunos son más bien generales y otros específicos, pero todos deben ser relevantes para las personas interesadas en la investigación. A veces, es preferible fijar sólo objetivos generales y determinar los específicos durante la marcha, para no buscar metas que quizá resulten triviales o imposibles. El objetivo puede ser muy preciso, como, por ejemplo, clarificar tal o cual fenómeno o área problemática, aun cuando sus problemas específicos o dificultades propias estén todavía muy enredados y sólo se puedan plantear o formular expresamente cuando la investigación esté más adelantada (pp. 130-131).

En lo relacionado con el diseño metodológico, este debe contemplar básicamente el paradigma de investigación, el enfoque y el o los métodos aplicados, la población y muestra (unidad de análisis y unidad de trabajo), las técnicas de recolección de información, el diseño o la elección de instrumentos para recoger datos, según el caso, ya que existen instrumentos previamente elaborados; el ingreso, permanencia y saldo del trabajo de campo; las técnicas de sistematización de los datos, y las técnicas de interpretación de los resultados, ya que se trata del "conjunto de procedimientos (métodos y técnicas) que se aplican para responder al problema de investigación y comprobar la hipótesis planteada" (Alva, 2002, p. 3).

En cuanto a la investigación cualitativa, se asume que ha alcanzado un estatus paradigmático, sobre la base de su enfoque epistemológico vivencialistaexperiencialista, el mismo que esencialmente postula la construcción de un conocimiento localizado a partir del acercamiento natural del investigador a la realidad y la comprensión e interpretación de esta, desde su experiencia vivida. Esta investigación por su naturaleza se realiza en el trabajo de campo donde el investigador, establece relaciones abiertas con los informantes clave, en ese sentido Taylor y Bogdan (1987), señalan:

El trabajo de campo incluye tres actividades principales. La primera se relaciona con una interacción social no ofensiva: lograr que los informantes se sientan cómodos y ganar su aceptación. El segundo aspecto trata sobre los modos de obtener datos: 
estrategias y tácticas de campo. El aspecto final involucra el registro de los datos en forma de notas de campo escritas (p. 5).

Se destaca entonces que el investigador cualitativo, realiza su labor en el propio lugar de los hechos, como afirman varios investigadores "en el terreno natural" donde los seres humanos interactúan libre y espontáneamente, en función de sus necesidades, intereses y motivaciones, por lo que no se requiere adaptar, adecuar, preparar o manipular las condiciones para desarrollar investigaciones con valor científico.

¿Cuáles son los criterios que justifican la selección de las técnicas y el diseño de instrumentos para recoger datos en la investigación cualitativa?

La rigurosidad en la selección de técnicas y en el diseño y aplicación de instrumentos de recolección de datos, determinan en cierta medida la validez científica de la investigación. Como afirma Torres (1996) citado por Hernández (2012), "la confiabilidad se asocia con la selección de informantes típicos, neutralizando creencias, conocimientos e imaginarios previos; la validez se asocia con procedimientos e instrumentos empleados para llegar a conclusiones válidas" (p. 8). Por tanto, la confiablidad y la validez de los instrumentos de recolección de datos, es altamente relevante en la investigación cualitativa. Cabe señalar que, al desarrollar la referida investigación en torno al hecho social educativo, se requiere de amplia experticia en el campo de la educación como una institución de la sociedad y para la sociedad.

Las técnicas e instrumentos recogen datos sobre los temas y subtemas de las categorías de análisis (iniciales o previas), como se dijo en líneas anteriores, durante el desarrollo de la investigación, pueden emerger otras categorías de análisis. Valles (2000), señala: "la función de los datos cualitativos se circunscribe a la fase exploratoria de una investigación, en la que los datos cuantitativos asumen la verificación de hipótesis teóricas ("objetivo más importante del proceso científico") ( $p$. 18).

El investigador cualitativo es sensible a los efectos causados por sí mismo sobre las personas que conforman el objeto de estudio, si bien, los efectos de la acción investigativa son difíciles de evitar, el investigador, intenta por todos los mecanismos posibles reducirlos, controlarlos y tenerlos en cuenta, al momento de interpretar los datos. Quecedo y Castaño (2002), afirman: "el investigador cualitativo, suspende o aparta sus creencias, opiniones y predisposiciones" (p. 5), a fin de conservar la necesaria independencia con respecto a los datos que está obteniendo y garantizar la veracidad de estos, lo cual no implica necesariamente apartar o abandonar las posibilidades de comprensión e interpretación del investigador cualitativo. González (2016, p. 8) citado por Ascorra y López (2016), refriéndose a las características inherentes de la investigación cualitativa, señala que son: "el carácter constructivo interpretativo del conocimiento; el carácter dialógico de la investigación cualitativa y la definición de lo singular como instancia legítima para la producción del conocimiento científico" (p. 2).

En la investigación objeto de este trabajo de reflexión, los métodos no son reductibles a pasos o etapas, la aplicación de estos sigue directrices generales que orientan el accionar del investigador, es decir, los métodos, técnicas e instrumentos que se emplean en la investigación cualitativa no son rígidos a pasos lógicos, lo cual, tiene concordancia con los planteamientos de varios autores, entre ellos Padrón (2007), quien, refiriéndose a la investigación cualitativa, señala: 
[..] es imposible manejar cualquier tesis epistemológica sin considerar la perspectiva preteórica desde la cual fue planteada y por eso las discusiones entre perspectivas diferentes (como es el caso entre realismo e idealismo, por ejemplo) resultan lógicamente irresolubles: al estar condicionadas por esos filtros o perspectivas cuyo carácter es precognitivo (pre-racional y pre- lógico) resultan irreductibles en un plano lógico y racional (p. 3).

Por consiguiente, el investigador emplea e incluso adapta las referidas herramientas metodológicas a las necesidades del contexto y no se esclaviza ante estos. De ahí, que el investigador tendrá la tarea de escoger (existen instrumentos de recolección de datos validados) o diseñar los instrumentos más adecuados, para obtener datos válidos y configurar un sistema de información confiable, que se corresponda con el objeto de estudio, en este caso particular con las exigencias del hecho social educativo. En tal sentido, Schettini y Cortazzo (2015), señalan que en: "la investigación cualitativa se identifican varios obstáculos en este proceso. El primero, que surge al trabajar con casos casi únicos, es el de la representatividad, obviamente, en la medida en que se pueda relacionar las características de un caso con una muestra aumentará la confianza en la investigación, en otros casos podrá interesar la investigación en su singularidad", en cuyos casos los instrumentos de recolección de datos no podrán ser aplicados de manera generalizada, más bien, deben ser escogidos o diseñados en función de la particularidad de lo que se investiga.

\section{CONCLUSIONES}

En varios estudios se encuentra que, históricamente, la investigación cualitativa fue considerada como parte de la investigación cuantitativa o paradigma positivista, ello se demuestra, ya que investigadores de reconocido prestigio reportaron hallazgos de investigaciones desarrolladas a través de la técnica de observación participante, pero lo hacían en términos altamente estadísticos. Teniendo en cuenta que la investigación cualitativa se instaura como un paradigma sobre las bases del enfoque epistemológico vivencialista-experiencialista, y que esta investigación al tener un carácter trans-disciplinar y multi-metódico, es ampliamente aplicada en el campo de las Ciencias Sociales, se destaca como la alternativa epistemológica idónea para la construcción del conocimiento científico a partir del estudio del hecho social educativo.

La escogencia de las técnicas y el diseño de instrumentos de recolección de datos deben tener en cuenta la naturaleza de la investigación, el objeto del estudio, el marco de referencia inicial, el contexto en el cual se investiga, los objetivos y el diseño metodológico, para garantizar la recolección de datos validos de la realidad estudiada como base para la entrega de resultados investigativos científicamente plausibles. Se destaca que el investigador cualitativo tiene una alta responsabilidad al momento de escoger las técnicas y al diseñar los instrumentos de recolección de datos, ya que ello en cierta medida incidirá en la calidad de datos recogidos como insumos para arribar a las conclusiones.

Una adecuada aplicación de la investigación cualitativa en el hecho social educativo debe ofrecer resultados creíbles (válidos) para los grupos humanos y no centrarse únicamente en los intereses y necesidades del investigador o de las instituciones que orientan ciertos procesos investigativos. Nótese que el investigador cualitativo, tiene un compromiso social inexcusable con los actores del contexto en el que realiza su labor investigativa, por lo que está obligado a ofrecer garantías suficientes de que los resultados que está exhibiendo se alcanzaron a través de la 
ejecución de procesos investigativos sólidos desde una visión científica, lo cual, eleva el nivel de credibilidad $y / 0$ plausibilidad de sus aportes en el desarrollo del conocimiento cualitativo.

\section{REFERENCIAS BIBLIOGRÁFICAS}

Alva A. (2002). Diseño metodológico. Lima: Universidad Nacional Mayor de San Marcos.

Arroyo Gonçalves, C. M. (2006). Modos de investigar los fenómenos sociales. Punto Cero, 11(12), 35-42.

Ascorra, P., \& López, V. (2016). Investigación cualitativa en subjetividad.

Psicoperspectivas [online], 15, (1), pp.1-4.

Campoy, T. J. \& Gomez, E. (2011). Capítulo 10: Técnicas e instrumentos cualitativos de recogida de datos. En Manual básico para la realización de tesinas, tesis y trabajos de investigación (pp. 273-300).

Casilimas, C. A. S. (1996). Investigación cualitativa. Bogotá: ICFES.

Durkheim, E. (2001). Las reglas del método sociológico. http://150.162.242.35/bitstream/handle/praxis/76/As\%20Regras\%20do\%20M\%C3\%A 9todo\%20Sociol\%C3\%B3gico.pdf?sequence=1\&isAllowed=y

Gurdián Fernández, A. (2010). El paradigma cualitativo en la investigación socio educativa. San José de Costa Rica: INIE.

Hernández, I. (2012). Investigación cualitativa: una metodología en marcha sobre el hecho social. Rastros Rostros, 14(27), 57-68.

Martínez, M. (2014). La investigación cualitativa (síntesis conceptual). Revista de Investigación en Psicología, 4(1), p. 123-146.

Mella, O. (1998). Naturaleza y orientaciones teórico-metodológicas de la investigación cualitativa. Santiago: CIDE, 51.

Padrón, J. (2007). Tendencias epistemológicas de la investigación científica en el siglo XXI. Cinta de Moebio, (28), 1-28

Peña, A. Q. (2006). Metodología de investigación científica cualitativa. Psicología: Tópicos de actualidad. LIMA: UNMSM, 47-84.

Quecedo Lecanda, R., \& Castaño Garrido, C. (2002). Introducción a la metodología de investigación cualitativa. Revista de psicodidáctica, (14), 5-39.

Flores, J. G., Gómez, G. R., \& Jiménez, E. G. (1999). Metodología de la investigación cualitativa. Málaga, Aljibe.

Scribano, A. (2000). Reflexiones epistemológicas sobre la investigación cualitativa en ciencias sociales. Cinta de Moebio, (8), 1-11.

Taylor, S. J., y Bogdan, R. (1987). Introducción a los métodos cualitativos de investigación (Vol. 1). Barcelona: Paidós.

Tejedor, E. Javier. (1986). La estadística y los diferentes paradigmas de investigación. Educar, (10), 079-101.

Usategui Basozabal, E. (2004). Durkheim: Conflicto y Educación. Cuestiones Pedagógicas, 2004-2005, (17): 277-290.

Valles, M. S. (2000). Técnicas cualitativas de investigación social. Síntesis Editorial.

López, F. y Salas, H. 2009. La Investigación Cualitativa en Administración Cinta Moebio. 35:128-145.

Schettini, P., \& Cortazzo, I. (2015). Análisis de datos cualitativos en la investigación social. Editorial de la Universidad Nacional de La Plata (EDULP). 